\title{
Anatomic Study of Exposed Posterior Malleolus Using the Posterolateral Ankle Approach
}

\author{
Xin Ma* \\ Department of Orthopedics, Huashan Hospital, Fudan University, China
}

*Corresponding author: Xin Ma, Department of Orthopedics, Huashan Hospital, Fudan University, N0.12 Middle Wulumuqi Road, Jingan District, Shanghai, China Tel: +86-02152887136; Fax: +86-021-5288713; Email: maxinshhs@126.com

\section{Research Article}

Volume 5 Issue 1

Received Date: February 06, 2021

Published Date: March 03, 2021

DOI: $10.23880 /$ jobd-16000203

\section{Abstract}

We compared the effect of the posterolateral ankle approach on the exposed posterior malleolus and vascular nerves in order to reduce the probability of vascular nerve injury during surgical exposure. Five corpses were randomly allocated to incision $\mathrm{A}$ and B groups. The tip of the lateral malleolus was used as a starting point, while the lateral line of the Achilles tendon was used as the endpoint to its trisection. Using the two points near the side of the Achilles tendon, we drew two vertical horizontal lines to represent incisions A and B, then measured the horizontal distances between the tip of the lateral malleolus and incision $\mathrm{A}$ (a), the tip of the lateral malleolus and incision B (b), and the tip of the lateral malleolus and the midpoint of the sural nerve and small saphenous vein (c). We then exposed the fibula from the posterior portion of the peroneus brevis muscles, dissected the flexor pollicis longus from the posterior edge of the fibula, and used Vernier calipers to measure the maximum length and width of the exposed bone block. There was no statistically significant difference between distances (a) and (c), but there was a significant difference between distances (b) and (c). The length of the exposed posterior malleolus did not differ significantly between incisions A and B, but the width differed significantly. Exposing the posterior malleolus using an approach closer to the lateral Achilles tendon is less likely to injure the sural nerve and small saphenous vein and results in a larger exposed area and easier manipulation. Thus, this could be a better surgical treatment for ankle fractures.

Keywords: Anatomy; Posterior Malleolus; Poster lateral Ankle Approach

\section{Introduction}

Posterior malleolus fracture (PMF) accounts for 7-44\% of ankle fractures. The lack of proper fracture reduction may lead to early joint degeneration and poor ankle function [1,2]. One of the commonly used PMF classification systems is the Haraguchi classification. According to this system, PMFs are divided into three categories: type I (posterior lateral oblique fractures, 67\%), type II (medial extension fractures, 19\%), and type III (small bone fractures, 14\%) [3]. Open reduction and reliable internal fixation for intra-articular fractures is generally accepted, while debate persists about the surgical management of PMF [4-6]. Surgical approaches for ankle fractures generally include lateral, medial, posterolateral, and posteromedial incisions [7]. The success of the surgery depends on the patient's clinical features and the design of the surgical incision. The surgical approach to PMF is determined based on the fracture location, extent, orientation, and safety [8]. The four mentioned methods are those most commonly used in clinical practice. The current references are mostly according to patients' clinical symptoms and anatomical features of incision design, which is suitable and practical for clinical doctors' scientific research work. 
However, to provide better guidance regarding surgical approaches, here we conducted a comparative study of the exposed ankle surface of normal ankle joints via the posterolateral surgical approach and recorded the effect of different access approaches on the vascular nerves to determine how to reduce of surgical exposure difficulty and decrease vascular nerve damage risk.

\section{Material and Methods}

\section{Material}

A total of 10 ankle specimens were obtained from four adult fresh-frozen cadavers (two male, two female) and one adult male corpse fixed with formalin. All specimens were free of malformations, trauma, and fractures. The average male height was $1.72 \pm 0.48 \mathrm{~m}$, while the average female height was $1.58 \pm 0.23 \mathrm{~m}$.

\section{Surgical Instruments}

One surgical knife handle, 10 blades, one pair of tissue scissors, one pair of wire scissors, one pair of toothed tweezers, one pair of toothless tweezers, two sets of retractors, one Vernier caliper with an accuracy of $0.01 \mathrm{~mm}$, one ordinary soft ruler with an accuracy of $0.1 \mathrm{~cm}$, and one Japanese Canon camera were used in this study.

\section{Methods}

Anterolateral ankle approach: A 6-cm longitudinal incision was made between the lateral margin of the Achilles tendon and the trailing edge of the fibula and the skin was cut layer by layer with special attention paid to protect the superficial sural nerve and small saphenous vein.

Measurement method: The tip of the lateral malleolus was set as a starting point at the same level outside the Achilles tendon as the endpoint to its trisection, two points were identified near the side of the Achilles tendon, and two vertical horizontal lines were drawn to represent incisions $\mathrm{A}$ and $\mathrm{B}$. The left and right sides of each specimen were randomly assigned to receive incision A or B (Figure 1). The same horizontal distance was measured from the tip of the lateral malleolus to the midpoint of the sural nerve and small saphenous vein (c) (Figure 2), the distance between the tip of the lateral malleolus and incision A (a), and the distance from the tip of the lateral malleolus to incision B (b) (Figure 3). The fibula was exposed from the posterior area of the peroneus brevis muscles and the flexor pollicis longus was dissected from the posterior edge of the fibula. Vernier calipers are used to measure the maximum bone block length and width. Each specimen was measured by two individuals and the measurements were averaged to reduce the measurement error (Figure 4).

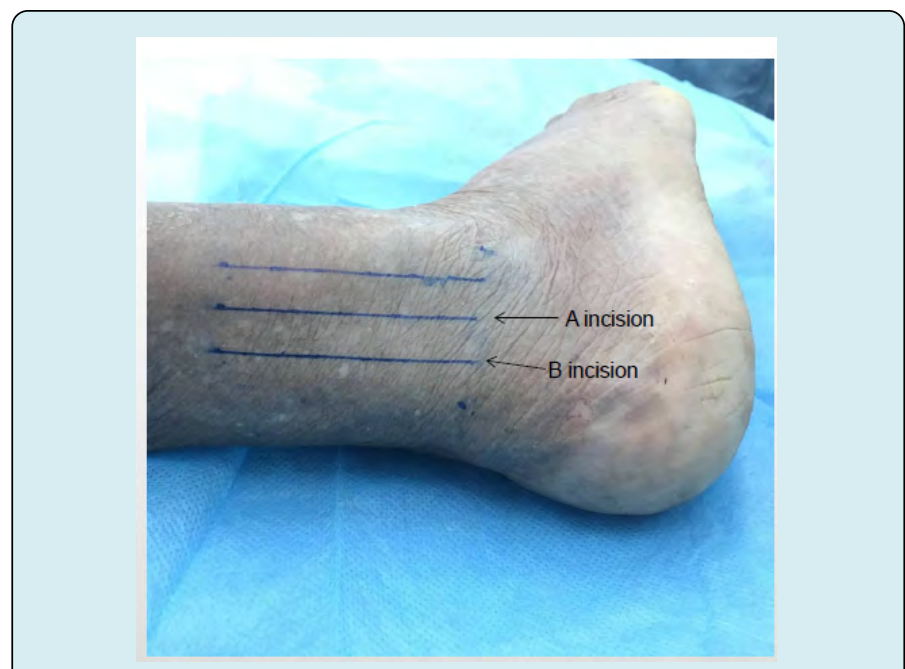

Figure 1: The tip of the lateral malleolus was set as a starting point at the same level outside the Achilles tendon as the endpoint to its trisection, two points were identified near the side of the Achilles tendon, and two vertical horizontal lines were drawn to represent incisions A and B.

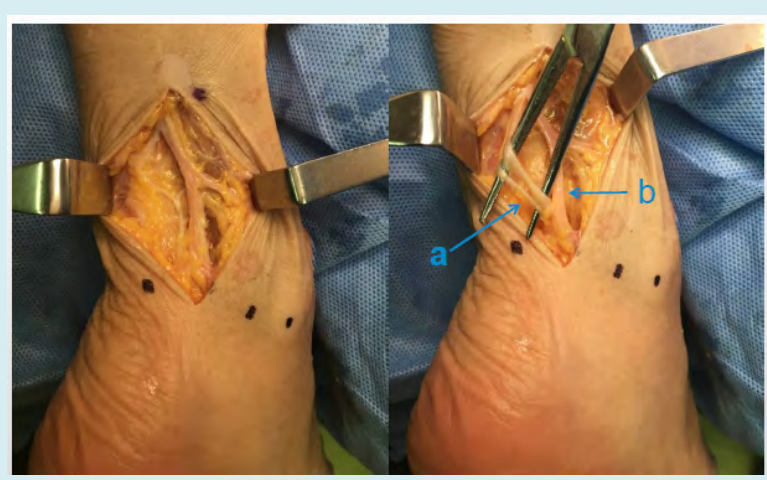

Figure 2: Sural nerve and small saphenous vein. a: sural nerve b: small saphenous vein.

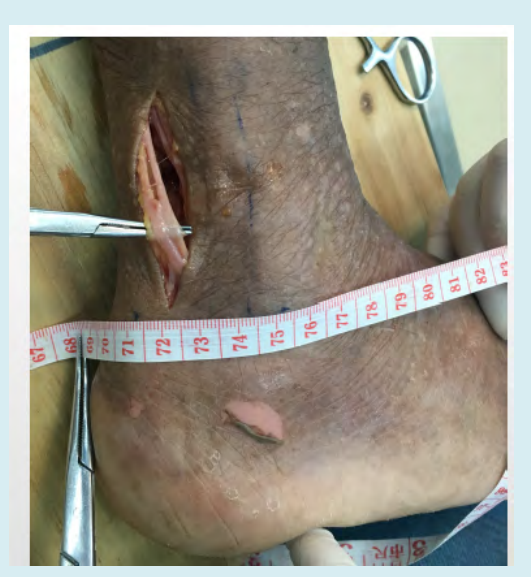

Figure 3: Average distance from the tip of the lateral malleolus to midpoint of sural nerve and small saphenous vein. 


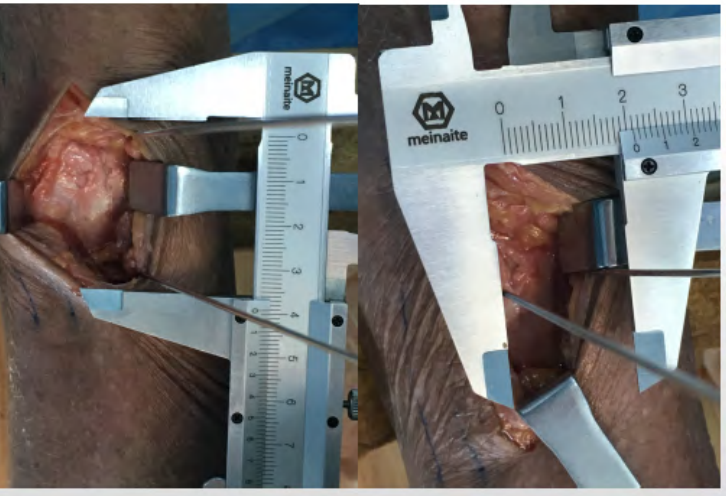

Figure 4: Measurement of length and width of exposed bone block.

\section{Statistical Analysis}

Anatomical measurements were made to obtain raw data. All of the test data are expressed as mean \pm standard deviation $(\mathrm{x} \pm \mathrm{s})$ in centimetres. T-test was performed using the SPSS 17.0 statistical software package, with $\alpha=0.05$ and $\mathrm{P}<0.05$ considered statistically significant.

\section{Results}

Distance from the tip of the lateral malleolus to incision A (a), incision B (b), or the midpoint of the sural nerve and small saphenous vein (c). There was no significant difference between distances (a) and (c) (P > 0.05), but a significant difference was observed between (b) and (c) $(\mathrm{P}<0.05)$ (Figure 5).

Length and width of ankle bone block exposed through incisions $\mathrm{A}$ and $\mathrm{B}$. The average length of the ankle bone block exposed through incision A was greater than that exposed through incision $\mathrm{B}$, but the difference was not significant $(\mathrm{P}$ $>0.05)$. The average width of the ankle bone block exposed through incision A was significantly lesser than that exposed through incision B $(\mathrm{P}<0.05)$ (Figure 6).
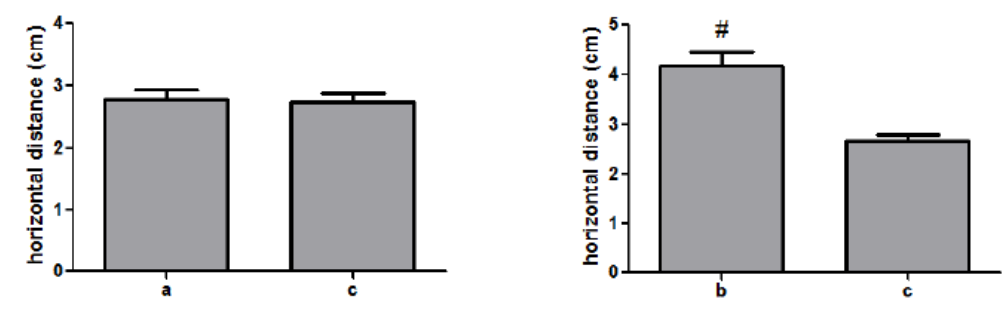

Figure 5: a: The horizontal distances between the tip of the lateral malleolus and incision A, b: The tip of the lateral malleolus and incision B, c: The tip of the lateral malleolus and the midpoint of the sural nerve and small saphenous vein, \#significant difference relative to $\mathrm{c}(\mathrm{P}<0.05)$.
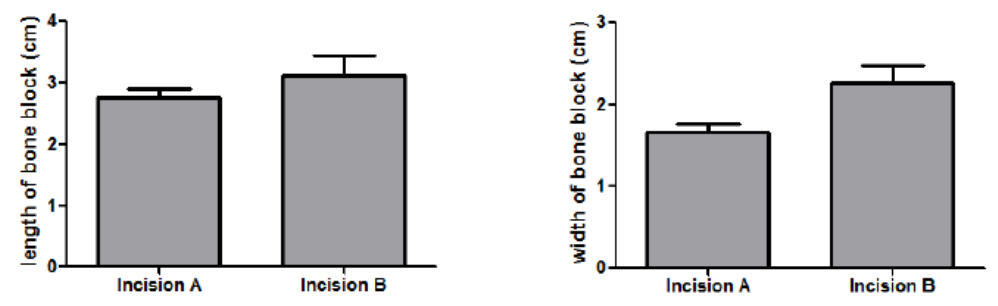

Figure 6: Length and width of exposed bone block, *significant difference relative to incision A $(\mathrm{P}<0.05)$.

\section{Discussion}

Ankle-related fracture, one of the most common intraarticular fractures that poses a high risk for young people, accounts for $14-44 \%$ of all ankle fractures [9]. The posterior malleolus, also known as the Volkmann nodule, forms the posterior lateral portion of the tibiotalar joint and is connected to the fibula by the posterior tibiofibular ligament 


\section{Journal of Orthopedics \& Bone Disorders}

[10-12]. Ankle fractures are often accompanied by internal and external malleolus fractures, which make the ankle joint unstable and prone to articular cartilage degeneration [13]. The incidence of traumatic arthritis of the ankle is significantly higher than the risk of ankle fractures and leads to poor functional foot recovery and prognosis [14]. The choice of the surgical approach used for ankle fractures is based on the following considerations: whether the lateral and lateral malleoli require open reduction at the same time; and internal fixation method $[15,16]$. The condition of the soft tissue surrounding the proposed incision affects a surgeon's ability to directly observe the fracture [17].

Olerud, et al. [18] suggested that ankle fracture fixation should be performed in the order of posterior malleolus, lateral malleolus, and medial malleolus, primarily because of the clear and safe exposure of the posterior malleolus and convenience and feasibility of fixation using the posterolateral incision. Talbot, et al. [19] reported in detail on the surgical procedure used to manage PMF, i.e., make a longitudinal incision in the posterior margin of the fibula, retract the tendon of the fibula to the inside to expose the posterior malleolus in a fixed order of lateral and posterior malleoli, and then make a medial incision to fix the medial malleolus. The main advantage of this approach is that it enables the direct visualization of the fractured bone block to facilitate the treatment of the posterolateral ankle fractures and is more conducive to post-anatomical reduction. With the help of gravity reduction and use of steel plate fixation, ankle fractures consist of osteochondral fragments; thus, a talus cartilage injury or compression injury could be more easily managed using a posterolateral approach [20].

Combined with the relevant literature, this anatomical study found that incision A was relatively closer to the lateral malleolus than incision B, similar to the lateral ankle approach used in clinical work. This incision was also closer to the sural nerve and small saphenous vein, which increases the risk of intraoperative injury [21]. Incision B was similar to the Achilles tendon lateral incision, which was positioned away from the vessels and nerves [22]. A comparison of the length and width of the posterior malleolus exposed through the two incisions revealed no significant differences in length, suggesting that the results may relate to the length of the incision. Since incision B was next to the posterior median line and resulted in greater exposure, the width of the exposed posterior malleolus was greater in this case than in case of incision A. Thus, the use of incision B could aid in fracture reduction and in ensuring upright positioning of hollow compression screws.

\section{References}

1. Odak S, Ahluwalia R, Unnikrishnan P, Michael Hennessy,
Simon Platt (2016) Management of posterior malleolar fractures: A systematic review. J Foot Ankle Surg 55(1): 140-145.

2. Fitzpatrick DC, Otto JK, McKinley TO, Marsh JL, Brown TD (2004) Kinematic and contact stress analysis of posterior malleolus fractures of the ankle. J Orthop Trauma 18(5): 271-278.

3. Haraguchi N, Haruyama H, Toga H, Fumio Kato (2006) Pathoanatomy of posterior malleolar fractures of the ankle. J Bone Joint Surg Am 88(5): 1085-1092.

4. Harper MC, Hardin G (1988) Posterior malleolar fractures of the ankle associated with external rotationabduction injuries. Results with and without internal fixation. J Bone Joint Surg Am 70(9): 1348-1356.

5. Miller AN, Carroll EA, Parker RJ, Helfet DL, Lorich DG (2010) Posterior malleolar stabilization of syndesmotic injuries is equivalent to screw fixation. Clin Orthop Relat Res 468(4): 1129-1135.

6. Streubel PN, McCormick JJ, Gardner MJ (2011) The posterior malleolus: should it be fixed and why? Curr Orthop Prax 22(1): 17-24

7. Gardner MJ, Streubel PN, Mccormick JJ, Sandra E Klein, Jeffrey E Johnson, et al. (2011) Surgeon Practices Regarding Operative Treatment of Posterior Malleolus Fractures J Foot \& ankle international 32(4): 385-393.

8. Mingo-Robinet J, López-Durán L, Galeote JE, Carmen Martinez-Cervell (2011) Ankle fractures with posterior malleolar fragment: management and results. J Foot Ankel Surg 50(2): 141-145.

9. Kellam J (1996) Fracture and dislocation compendium. J Orthop Trauma 10(S1): 1-155.

10. Macko VW, Matthews LS, Zwirkoski P, Goldstein SA (1991) The joint-contact area of the ankle. The contribution of the posterior malleolus. J Bone Joint Surg Am 73(3): 347-351.

11. Scheidt KB, Stiehl JB, Skrade DA, Barnhardt T (1992) Posterior malleolar ankle fractures: an in vitro biomechanical analysis of stability in the loaded and unloaded states. J Orthop Trauma. 6(1): 96-101.

12. Miller AJ (1974) Posterior malleolar fractures. J Bone Joint Surg Br 56B(3): 508-512.

13. Patel A, Charles L, Ritchie J (2016) A Complication of Posterior Malleolar Fracture Fixation. J Foot Ankle Surg 


\section{Journal of Orthopedics \& Bone Disorders}

55(2): 383-386.

14. Forberger J, Sabandal PV, Dietrich M, Jan Gralla, Thomas Lattmann, et al. (2009) Posterolateral approach to the displaced posterior malleolus: functional outcome and local morbidity. Foot Ankle Int 30(4): 309-314.

15. Shah NH, Sundaram RO, Velusamy A, Braithwaite IJ (2007) Five-year functional outcome analysis of ankle fracture fixation. Injury 38(11): 1308-1312.

16. Amorosa LF, Brown GD, Greisberg J (2010) A surgical approach to posterior pilon fractures. J Orthop Trauma 24(3): 188-193.

17. Abdelgawad AA, Kadous A, Kanlic E (2011) Posterolateral approach for treatment of posterior malleolus fracture of the ankle. J Foot Ankle Surg 50(5): 607-611.

18. Olerud P, Ahrengart L, Ponzer S, Jenny Saving, Jan Tidermark (2011) Internal fixation versus nonoperative treatment of displaced 3-part proximal humeral fractures in elderly patients: a randomized controlled trial. Journal of Shoulder and Elbow Surgery 20(5): 747755.

19. Talerbot M, Steenblock TR, Cole PA (2005) Posterolateral approach for open reduction and internal fixation of trimalleolar ankle fractures. Can J Surg 48(6): 487-490.

20. Jaskulka RA, Ittner G, Schedl R (1989) Fractures of the posterior tibial margin: their role in the prognosis of malleolar fractures. J Trauma 29(11): 1565-1570.

21. De Vries JS, Wijgman AJ, Sierevelt IN, Schaap GR (2005) Long-term results of ankle fractures with a posterior malleolar fragment. J Foot Ankle Surg 44(3): 211-217.

22. Webb J, Moorjani N, Radford M (2000) Anatomy of the sural nerve and its relation to the Achilles tendon. Foot Ankle Int 21(6): 475-477. 